\title{
Shortening of Leucocyte Telomerase Length Is Independently Correlated With High Body Mass Index and Subcutaneous Obesity (Predominantly Truncal), in Asian Indian Women With Prediabetes
}

\section{Surya Prakash Bhatt}

Department of Pulmonary, Critical Care and Sleep Medicine,All India Institute of Medical Sciences, Ansari Nagar, New Delhi 110029, India

Anoop Misra ( $\square$ anoopmisra@gmail.com )

Fortis C-DOC Center of Excellence for Diabetes, Metabolic Diseases, and Endocrinology, B-16, Chirag Enclave, New Delhi, India

\section{Ravindra Mohan Pandey}

Department of Biostatistics, All India Institute of Medical Sciences, Ansari Nagar, New Delhi 110029, India

\section{Ashish Datt Upadhyay}

Department of Biostatistics, All India Institute of Medical Sciences, Ansari Nagar, New Delhi 110029, India

\section{Research Article}

Keywords: Leucocyte telomerase length, aging, prediabetes, obesity, Asian Indians

Posted Date: September 9th, 2021

DOI: https://doi.org/10.21203/rs.3.rs-876526/v1

License: (c) (1) This work is licensed under a Creative Commons Attribution 4.0 International License. Read Full License 


\section{Abstract}

Introduction: Leucocyte telomerase length $(\mathrm{LTL})$ are inked to accelerate aging and premature mortality. In this research, we aimed to explore the relations between biochemical and anthropometry markers and LTL in Asian Indian women with prediabetes.

Methods: In this study, 797 prediabetic women (aged 20-60 years, obese, 492; non obese, 305) were recruited. Demographic and clinical profiles, medical history, skin exposure and duration of sunlight exposure were determined. Anthropometry, fasting blood glucose and serum 25-hydroxyvitamin $D$ $[25(\mathrm{OH}) \mathrm{D}]$ were evaluated. LTL was quantified by a quantitative polymerase chain reaction (qPCR). The study subjects were separated into quartiles groups according to the LTL.

Results: The average telomerase length (T/S) ratio was significantly decreased with increasing age. The average telomerase length (T/S) ratio was significantly shorter in obese women with prediabetes $(p<0.05)$. Univariate and multivariable linear regression analysis after adjustment for age, family income, education and hypertension showed that LTL was inversely correlated with body mass index (BMI), waist and hip circumference, waist-hip and waist-to-height ratio, and truncal skinfolds (subscapular, lateral thoracic, and subscapular/triceps ratio, central and total). Multivariable linear regression analysis identified BMI $(93 \%, p<0.0001)$, central $(92 \%, p<0.0001)$ and total skinfolds $(90 \%, p<0.0001)$ as independent predictors of LTL.

Conclusions: Besides age, obesity, and subcutaneous adiposity (predominantly truncal) are major contributors to telomerase shortening in Asian Indian women with prediabetes.

\section{Introduction}

Prediabetes and type 2 diabetes mellitus (T2DM) are increasing in developing countries (1). South Asians develop diabetes a decade before and at a lower body mass index (BMI) than White population, have prominent abdominal obesity, and accelerated conversion from prediabetes to diabetes (2). Importantly, India has one of the maximum number of people with pre-diabetes and diabetes(3).

Diabetes can have serious health implications that affect life expectancy. The United Kingdom diabetes study stated a 5 years decrease of life expectancy for men aged 45 to 50 years at the time of diagnosis of diabetes when compared to the common population (4). Data from the Emerging Risk Factor Collaboration likewise presented that life years lost were significantly larger when diabetes is present at earlier ages (5). A Scotland-wide study from German administrative claims data (n, 2,900,065) showed that T2DM is interrelated to lower life expectancy regardless of an individual's socioeconomic status. (6). There are no published data regarding prediabetes and shorting of lifespan in Asian Indians.

Telomerase is an important enzyme that maintains leukocyte telomerase length (LTL) and cellular replicate potential. LTL naturally shortens with each cell phase, and cells with critically short telomerase undergo replicative senescence and apoptosis which promote organismal aging and age-related 
diseases. LTL shortening is accelerated by oxidative stress, inflammation, and cell production (7). The level of leukocyte telomerase activity is important in determining LTL in aging cells and tissues (8). Given that telomerase, the enzyme responsible for maintaining telomerase lengths, is not expressed at levels sufficient to prevent telomerase shortening in most of our cells, it progressively erodes with advancing age (9). Short LTL in peripheral-blood mononuclear cells (PBMCs) is linked with ageing and its related diseases, such as obesity, T2DM and cardiovascular disease (10). Some lifestyle interventions, including Ornish and the Mediterranean diets, have demonstrated decreased leukocyte telomerase activity (11)

There is paucity of data regarding LTL activity and telomerase length in individuals with prediabetes. It is important to note that effects of pistachio intake on LTL has been shown only one previous study, in which limited number of Spanish subjects $(n, 49)$ having prediabetes were recruited $(12)$. Association between LTL, prediabetes and obesity in Asian Indians has not been researched. To better highlight this knowledge gap, we proposed to investigate the relationship of LTL in prediabetes with obesity in community dwelling Asian Indian women residing in North India in New Delhi.

\section{Methods}

Subject Recruitment: We directed a cross-sectional population-based study from July 2015 to March 2020. The study was sanctioned by the institutional ethics committee. Study subjects were randomly designated to have approximate representation from each income group (higher 10\%, middle 65$70 \%$, and lower $\sim 15-20 \%$ ) from forty-one residential sites in Delhi. Subjects with pregnancy, severe end organ damage or chronic diseases, malignancy, and known T2DM and other endocrine illnesses were excluded from the study. After informed consent, 797 females, 20-60 years of age, were enrolled in this study. The institutional and licensing committee approved all experiments and it is also confirmed that all experiments were performed in accordance with relevant guidelines and regulations.

Demographic and Clinical Profiles: Demographic and clinical profiles, medical history (personal and family), socioeconomic features, skin exposure and time of sunlight exposure were recorded with the use of pre-validated questionnaire. Skin exposure was measured as percentage of body surface area (face/hands, face/hands and arms, and face/hands and legs) exposed to sunlight. The interval of sun exposure (minutes/day) was evaluated in the following mode; $<5$ minutes, 5-15 minutes, 15-30 minutes and $>30$ minutes. Blood pressure was documented by a standard mercury sphygmomanometer, over the right arm in sitting situation.

Anthropometric Measurements: BMI, circumferences [waist circumference (WC) and hip circumference $(\mathrm{HC})]$ and skinfold thickness at 6 sites (biceps, triceps, anterior axillary, suprailiac, subscapular and lateral thoracic) was recorded (13). Waist-hip ratio (WHR) and waist-height ratio (WhtR) was calculated. Sum of all skinfolds ( $\Sigma 6 \mathrm{SF}$, total skinfolds), ratios of subscapular and triceps skinfolds (SS/TR ratio), central skinfolds (sum of subscapular and suprailiac) and peripheral skinfolds (sum of biceps and triceps) were also calculated. 
Biochemical Analysis: Venous blood sample was collected into vacutainer tubes containing plain and EDTA vials. The fasting blood sample was processed into different aliquots (including whole blood and blood clot) within 2hours into $1.0 \mathrm{~mL}$ FluidX tubes (FluidX, Cheshire, UK) and frozen at $-80^{\circ} \mathrm{C}$ freezer (Thermo Fisher Scientific, Waltham, MA, USA).

Fasting blood glucose (FBG) and serum 25-hydroxy vitamin D [25(OH) D] levels were analysed as previously described (13). The intra-assay coefficient of variation of $25(\mathrm{OH}) \mathrm{D}$ was $1.81 \%$ and the coefficient of inter-assay was $2.34 \%$.

DNA Isolation and Quantification: DNA was separated from peripheral blood mononuclear cells using the QIAamp DNA extraction kit (Qiagen, Hilden, Germany) and stored at $-20^{\circ} \mathrm{C}$ for the future experiments (14). After DNA isolation, the DNA samples were quantified and diluted to $50 \mathrm{ng} / \mu \mathrm{L}$. The concentration and quality of DNA were both measured by using a nanodrop (Nanodrop Technologies, Wilmington, NC, USA) and samples included for analysis all had an optical density ratio A 260/A280 > 1.8.

Measurement of Leukocyte Telomerase Length: LTL was analysed with a quantitative polymerase chain reaction (qPCR) based technique that compares telomerase repeat sequence copy number ( $T$ ) to a reference single copy-gene copy number $(S)$ as previously described $(15,16)$. The telomerase length for each sample was estimated using the telomerase to single copy gene ratio (T/S ratio) with the calculation of $\Delta \mathrm{Ct}\left[\mathrm{Ct}{ }^{\text {(telomere) }} / \mathrm{Ct}{ }^{\text {(single gene) }}\right]$. $\mathrm{T} / \mathrm{S}$ ratio for each sample $(\mathrm{x})$ was normalized to the mean $\mathrm{T} / \mathrm{S}$ ratio of the reference sample $\left[2^{-(\Delta \mathrm{Ctx}-\Delta \mathrm{Ctr})}=2^{-\Delta \Delta \mathrm{Ct}}\right]$, which was used for the standard curve, both as a reference sample and as a validation sample. The Measurement consists of determining the relative ratio (T/S ratio) of $n$ g of telomerase $(T)$ to $n g$ of albumin (single-copy gene, $S$ ) in experimental samples using a standard curve. The T/S ratio is proportional to the average telomerase length. All qPCR assay was performed using filtered pipette tips to prevent amplification of contaminants. Reactions were set up on ice to prevent DNA polymerase activity, non-specific amplification and to minimize potential primerdimerization. All analyses were done blinded to cross sectional status of the individual.

The coefficient of variations (CVs) of the inter-plate T/S were $11.6 \%$, and $12.2 \%$ for the long and short telomerase QC samples, respectively. Inter- and intra-plate CVs of calibrator DNA samples were $10.2 \%$, and $8.3 \%$, respectively. Mean ratio of long to short telomerase QC samples in our assays was 3.9 with $4.5 \% \mathrm{CV}$. All samples in our study were assayed in triplicate, and the results were consistent. Less than $12 \%$ of samples had a T/S CV more than $10 \%$.

\section{Definitions}

Overweight and obesity were defined as BMI $23-24.9 \mathrm{~kg} / \mathrm{m}^{2}$ and $>25 \mathrm{~kg} / \mathrm{m}^{2}$, respectively (17). Abnormal blood pressure was $\geq 130 / 85 \mathrm{mmHg}$. Prediabetes was defined as FBG levels $\geq 100$ and $125.9 \mathrm{mg} / \mathrm{dl}$. Serum $25(\mathrm{OH})$ D status was defined as deficient $(<10 \mathrm{ng} / \mathrm{ml}]$, insufficient $[10.1-30 \mathrm{ng} / \mathrm{ml})$ or sufficient $[30.1-100 \mathrm{ng} / \mathrm{ml})(18)$. LTL were categorised in quartiles, $0-25$ th percentile (1st quartile; LTL ratio < 0.83 ), 26th - 50th percentile (2nd quartile, LTL ratio 0.84-0.87), 51st - 75th percentile (3rd quartile, LTL ratio 
0.88-0.93), and 76th - 100th percentile (4th quartile, LTL ratio 0.94-0.98). Because several values of LTL were gathered around cut-off values of quintiles, slightly changed numbers of subjects were separated in each quintile.

\section{Statistical Analysis}

Complete data were entered in an Excel worksheet (Microsoft Corp, Washington, USA). The distribution of demographic, clinical profiles, socioeconomic, medical history (personal and family), behavioral characteristics, sun and skin exposure and biochemical profiles were confirmed for estimated regularity. Mean and standard deviation and number (\%) was used to summarize the variables. Relationships between LTL and various indices of body composition were identified by Pearson correlation analyses. Associations with LTL were estimated using partial correlations that adjusted by age. Association of categorical variables were assessed by chi-square/Fisher exact test. The continuous variables were compared between obesity by independent t-test/Wilcoxon rank sum test, as appropriate. The comparisons of clinical, biochemical, anthropometry and body composition profiles among different LTL quartiles were performed by mean of ANOVA and Kruskal-Wallis test, as appropriate, and further trend was seen by non-parametric trend test. Univariate and multivariable linear regression analysis was used to find independent effect of LTL obesity marker after adjusting confounder. Complete data was analysed using Stata - 14 (LLC 4905 Lakeway Drive College Station, Texas 77845 - 4512. USA). For all above, p value of $<0.05$ was considered as statistically significant.

\section{Results}

\section{Clinical and Biochemical Profiles (Table 1 and 2)}

Educational status, employment status, family income, personal history of hypertension, family history of heart disease, skin exposure (face and hands) to sun were significantly higher in obese subjects with prediabetes as compared to those with non-obese with prediabetes. 
Table 1

Socio-economic profile, behavioral characteristics and skin and sun exposure

\begin{tabular}{|c|c|c|c|c|c|}
\hline Variables & & $\begin{array}{l}\text { Total } \\
(n, 797)\end{array}$ & $\begin{array}{l}\text { Obese } \\
(n, 492)\end{array}$ & $\begin{array}{l}\text { Non obese } \\
(n, 305)\end{array}$ & $\begin{array}{l}\mathrm{P} \\
\text { value }\end{array}$ \\
\hline \multirow[t]{2}{*}{ Religion } & Hindu & $\begin{array}{l}794 \\
(99.62)\end{array}$ & $\begin{array}{l}491 \\
(61.84)\end{array}$ & $\begin{array}{l}303 \\
(38.16)\end{array}$ & \multirow[t]{2}{*}{0.23} \\
\hline & Others & $03(0.37)$ & $01(33.3)$ & $02(66.7)$ & \\
\hline \multirow[t]{2}{*}{ Marital status } & Married & $\begin{array}{l}492 \\
(61.63)\end{array}$ & $\begin{array}{l}226 \\
(45.93)\end{array}$ & $\begin{array}{l}266 \\
(54.07)\end{array}$ & \multirow[t]{2}{*}{0.35} \\
\hline & Unmarried & $\begin{array}{l}305 \\
(38.37)\end{array}$ & $\begin{array}{l}225 \\
(73.78)\end{array}$ & $80(26.22)$ & \\
\hline \multirow[t]{7}{*}{ Education } & $\begin{array}{l}\text { Never attended } \\
\text { school }\end{array}$ & $\begin{array}{l}147 \\
(18.44)\end{array}$ & $56(38.10)$ & $91(61.90)$ & \multirow[t]{7}{*}{0.04} \\
\hline & Grade 1st to 8th & $\begin{array}{l}164 \\
(20.58)\end{array}$ & 59 (35.98) & $\begin{array}{l}105 \\
(64.02)\end{array}$ & \\
\hline & Grades 9th to 10th & $\begin{array}{l}110 \\
(13.80)\end{array}$ & $34(30.91)$ & 76 (69.09) & \\
\hline & Higher secondary & $\begin{array}{l}106 \\
(13.30)\end{array}$ & 38 (35.85) & $68(64.15)$ & \\
\hline & College/diploma & $23(2.89)$ & $4(17.39)$ & $19(82.61)$ & \\
\hline & Graduate & $\begin{array}{l}157 \\
(18.70)\end{array}$ & $65(41.40)$ & $92(58.60)$ & \\
\hline & Post graduate & $90(11.29)$ & )$^{49(53.41}$ & $41(46.59)$ & \\
\hline \multirow[t]{2}{*}{ Employment status } & Employed & $\begin{array}{l}454 \\
(56.96)\end{array}$ & $\begin{array}{l}293 \\
(64.54)\end{array}$ & $\begin{array}{l}161 \\
(35.46)\end{array}$ & \multirow[t]{2}{*}{0.05} \\
\hline & Housewife & $\begin{array}{l}343 \\
(43.03)\end{array}$ & $\begin{array}{l}198 \\
(57.23)\end{array}$ & $\begin{array}{l}145 \\
(42.77)\end{array}$ & \\
\hline \multirow[t]{3}{*}{ Family income } & Less than Rs 10000 & $\begin{array}{l}286 \\
(35.88)\end{array}$ & $\begin{array}{l}199 \\
(69.58)\end{array}$ & 87 (30.42) & \multirow[t]{3}{*}{0.04} \\
\hline & Rs 10001 - Rs 30000 & $\begin{array}{l}333 \\
(41.78)\end{array}$ & $\begin{array}{l}200 \\
(60.06)\end{array}$ & $\begin{array}{l}154 \\
(39.94)\end{array}$ & \\
\hline & More than Rs 30000 & $\begin{array}{l}178 \\
(22.34)\end{array}$ & $99(55.61)$ & 79 (44.39) & \\
\hline Tobacco consumption & & 04 & $2(0.4741)$ & $2(0.66)$ & 0.63 \\
\hline Alcohol consumption & & 02 & $2(0.41)$ & 0 & 0.52 \\
\hline
\end{tabular}

Values are given as the number, \%. P value is $<0.05$ is statistically significant 


\begin{tabular}{|c|c|c|c|c|c|}
\hline Variables & & $\begin{array}{l}\text { Total } \\
(n, 797)\end{array}$ & $\begin{array}{l}\text { Obese } \\
(n, 492)\end{array}$ & $\begin{array}{l}\text { Non obese } \\
(n, 305)\end{array}$ & $\begin{array}{l}\mathrm{P} \\
\text { value }\end{array}$ \\
\hline \multirow[t]{4}{*}{$\begin{array}{l}\text { Personal medical } \\
\text { history }\end{array}$} & Hypertension & $92(11.54)$ & $\begin{array}{l}70 \\
(714.23)\end{array}$ & $22(7.21)$ & 0.003 \\
\hline & Thyroid & $81(10.16)$ & $55(11.18)$ & $26(8.52)$ & 0.27 \\
\hline & Tuberculosis/ asthma & $8(1)$ & $4(0.81)$ & $4(1.31)$ & 0.49 \\
\hline & Skeletal bone disorder & $16(2.01)$ & $13(2.64)$ & $3(0.98)$ & 0.08 \\
\hline \multirow[t]{4}{*}{ Family medical history } & Obesity & $10(1.21)$ & $8(1.63)$ & $2(0.66)$ & 0.19 \\
\hline & Diabetes & $\begin{array}{l}148 \\
(18.57)\end{array}$ & $91(18.50)$ & 57 (18.69) & 0.51 \\
\hline & Hypertension & 90 (11.29) & $56(11.38)$ & $34(11.15)$ & 0.50 \\
\hline & $\begin{array}{l}\text { Coronary heart } \\
\text { disease }\end{array}$ & $37(4.64)$ & $29(5.89)$ & $8(2.62)$ & 0.02 \\
\hline \multirow[t]{2}{*}{ Skin exposure } & Face/hands & $\begin{array}{l}511 \\
(64.12)\end{array}$ & $\begin{array}{l}316 \\
(61.84)\end{array}$ & $\begin{array}{l}195 \\
(38.16)\end{array}$ & \multirow[t]{2}{*}{0.002} \\
\hline & Face/hands/arms/leg & $\begin{array}{l}286 \\
(35.88)\end{array}$ & $\begin{array}{l}176 \\
(61.54)\end{array}$ & $\begin{array}{l}110 \\
(38.46)\end{array}$ & \\
\hline \multirow[t]{3}{*}{$\begin{array}{l}\text { Duration of Sun } \\
\text { exposure }\end{array}$} & $<5$ minutes & $\begin{array}{l}102 \\
(12.79)\end{array}$ & $52(50.98)$ & $50(49.02)$ & \multirow[t]{3}{*}{0.03} \\
\hline & $5-15$ minutes & $\begin{array}{l}495 \\
(62.12)\end{array}$ & $\begin{array}{l}213 \\
(43.03)\end{array}$ & $\begin{array}{l}282 \\
(56.97)\end{array}$ & \\
\hline & $15-30$ minutes & $\begin{array}{l}200 \\
(25.09)\end{array}$ & $91(45.50)$ & $\begin{array}{l}191 \\
(54.50)\end{array}$ & \\
\hline
\end{tabular}


Table 2

Demographic, clinical, body composition and biochemical profiles.

\begin{tabular}{|c|c|c|c|c|}
\hline \multirow[t]{3}{*}{ Variables } & Total & Obese & Non obese & $P$ value \\
\hline & Mean \pm SD & Mean \pm SD & Mean \pm SD & \\
\hline & n, 797 & n, 492 & $\mathrm{n}, 305$ & \\
\hline Numbers (\%) & $797(100)$ & $492(61.73)$ & $305(38.27)$ & \\
\hline Age (Years) & $42.02 \pm 11.38$ & $43.73 \pm 10.29$ & $39.26 \pm 12.48$ & $\begin{array}{l}<.0001 \\
0.00\end{array}$ \\
\hline \multicolumn{5}{|l|}{ Blood pressure (mmHg) } \\
\hline Systolic & $\begin{array}{l}122.68 \pm \\
12.76\end{array}$ & $\begin{array}{l}124.95 \pm \\
11.75\end{array}$ & $\begin{array}{l}119.02 \pm \\
13.49\end{array}$ & $\begin{array}{l}<.0001 \\
0.00\end{array}$ \\
\hline Diastolic & $80.55 \pm 7.92$ & $82.28 \pm 7.40$ & $77.77 \pm 7.94$ & $\begin{array}{l}<.0001 \\
0.000\end{array}$ \\
\hline \multicolumn{5}{|l|}{ Body composition } \\
\hline Weight (Kg) & $61.86 \pm 11.96$ & $68.47 \pm 9.0$ & $51.20 \pm 7.71$ & $\begin{array}{l}<.0001 \\
0.00\end{array}$ \\
\hline Height (cm) & $153.49 \pm 5.90$ & $152.90 \pm 5.87$ & $154.36 \pm 5.86$ & 0.001 \\
\hline Body mass index $\left(\mathrm{Kg} / \mathrm{m}^{2}\right)$ & $26.36 \pm 3.83$ & $29.41 \pm 3.83$ & $21.42 \pm 2.72$ & $\begin{array}{l}< \\
0.0001\end{array}$ \\
\hline \multicolumn{5}{|c|}{ Circumferences $(\mathrm{cm})$ and ratios } \\
\hline Waist & $89.18 \pm 7.53$ & $93.82 \pm 4.42$ & $81.69 \pm 5.06$ & $\begin{array}{l}<.0001 \\
0.00\end{array}$ \\
\hline Hip & $96.34 \pm 7.93$ & $100.99 \pm 4.30$ & $88.80 \pm 6.62$ & $\begin{array}{l}<.0001 \\
0.00\end{array}$ \\
\hline \multicolumn{5}{|l|}{ Ratio } \\
\hline Waist-hip & $0.92 \pm 0.02$ & $0.93 \pm 0.008$ & $0.92 \pm 0.02$ & $\dot{c}_{0.0001}$ \\
\hline Waist-height & $0.58 \pm 0.05$ & $0.61 \pm 0.03$ & $0.53 \pm 0.38$ & $\begin{array}{l}<.0001 \\
0.000\end{array}$ \\
\hline \multicolumn{5}{|l|}{ Skinfolds and $(\mathrm{mm})$ ratios } \\
\hline Biceps & $27.09 \pm 6.23$ & $30.43 \pm 5.04$ & $25.71 \pm 3.67$ & $\begin{array}{l}<.0001 \\
0.000\end{array}$ \\
\hline Triceps & $28.63 \pm 5.88$ & $31.85 \pm 5.06$ & $23.44 \pm 2.35$ & $\begin{array}{l}< \\
0.0001\end{array}$ \\
\hline
\end{tabular}

Values are given as the mean \pm standard deviation. T/S ratio, telomere to single copy gene ratio. $\mathrm{P}$ value is $<0.05$ is statistically significant 


\begin{tabular}{|c|c|c|c|c|}
\hline \multirow[t]{3}{*}{ Variables } & Total & Obese & Non obese & $P$ value \\
\hline & Mean \pm SD & Mean \pm SD & Mean \pm SD & \\
\hline & n, 797 & n, 492 & n, 305 & \\
\hline Anterior axillary & $12.1 \pm 3.7$ & $14.0 \pm 4.0$ & $10.2 \pm 3.4$ & 0.04 \\
\hline Subscapular & $35.05 \pm 9.88$ & $40.07 \pm 9.58$ & $26.95 \pm 0.89$ & $<.0001$ \\
\hline Lateral thoracic & $36.41 \pm 11.3$ & $42.96 \pm 9.61$ & $25.85 \pm 2.73$ & $\begin{array}{l}<.0001 \\
0.001\end{array}$ \\
\hline Suprailliac & $37.68 \pm 11.55$ & $44.25 \pm 9.95$ & $27.07 \pm 2.53$ & $<.0001$ \\
\hline Subscapular/triceps ratio & $1.21 \pm 0.11$ & $1.24 \pm 0.12$ & $1.15 \pm 0.09$ & $<.0001$ \\
\hline Central & $72.73 \pm 21.35$ & $84.32 \pm 19.51$ & $54.02 \pm 3.33$ & $<.0001$ \\
\hline Peripheral & $56.26 \pm 11.99$ & $56.40 \pm 11.99$ & $56.05 \pm 12.09$ & 0.69 \\
\hline 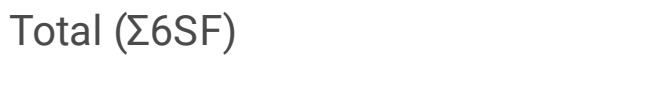 & $\begin{array}{l}176.96 \pm \\
48.58\end{array}$ & $\begin{array}{l}203.56 \pm \\
43.24\end{array}$ & $\begin{array}{l}139.22 \pm \\
15.57\end{array}$ & $\begin{array}{l}< \\
0.0001\end{array}$ \\
\hline \multicolumn{5}{|c|}{ Biochemical Investigations and Leukocyte Telomerase Length } \\
\hline Fasting blood glucose (mg/dL) & $100.1 \pm 7.54$ & $111.62 \pm 7.40$ & $101.3 \pm 7.76$ & 0.05 \\
\hline Vitamin D (ng/mL) & $\begin{array}{l}32.12 \pm 1 \\
1.79\end{array}$ & $28.33 \pm 12.2$ & $35.94 \pm 11.19$ & 0.04 \\
\hline $\begin{array}{l}\text { Leukocyte telomerase length (T/S } \\
\text { ratio) }\end{array}$ & $0.95 \pm 0.09$ & $0.85 \pm 0.02$ & $0.94 \pm 0.01$ & $<.0001$ \\
\hline
\end{tabular}

The mean \pm standard deviation for age was $42.02 \pm 11.4$ years. Mean age, systolic and diastolic blood pressure, weight, BMl, circumferences and ratios thereof (WC, HC, W-HR, W-htR), skinfold thickness and ratios thereof (biceps, triceps, subscapular, suprailiac, $\Sigma 6 \mathrm{SF}$, subscapular/triceps ratio, central and peripheral) and FBG levels were significantly increased in obese subjects with prediabetes as compared to those with non-obese with prediabetes. Serum $25(\mathrm{OH}) \mathrm{D}$ levels were significantly increased in nonobese subjects with prediabetes as compared to those with obese subjects with prediabetes.

\section{Total Leukocyte Telomerase Length (Table 4, Figs. 1 and 2)}

LTL was significantly decreased in older subjects as compared to younger subjects (Fig. 1). Mean values of LTL were significantly incerased in obese subjects with prediabetes as compared to those with non- 
obese with prediabetes (Fig. 2). After stratifying the sample according to LTL quartiles, we observed age, systolic and diastolic blood pressure, weight, BMI, circumferences and their ratios [WC, HC, W-HR, W-htR), skinfolds [biceps, triceps, subscapular, suprailiac, E6SF, subscapular/triceps ratio and central) and FBG levels were significantly increased in $I^{\text {st }}$ quartile as compared to other quartiles. Further, serum $25(\mathrm{OH}) \mathrm{D}$ levels were significantly higher in IVth quartile as compared to other quartiles.

\section{Partial Correlations (figures 3 \&4)}

We observed a significant partial correlation of LTL with subscapular/triceps ratio $[(r=-0.4371, p=$ $0.00001)$, central skinfolds $(r=-0.8375, p=0.0001), \Sigma 6 S F(r=-0.8560, p=0.00001)$, FBG $[(r=-0.1234$, $p=0.00001)$, serum 25(OH) D levels $(r=-0.078, p=0.024)$, BMI $(r=-0.8783, p=0.00001)$ and W-htR $(r=$ $-0.1672, p=0.0001)$, adjusted by age.

\section{Univariate and Multivariable Linear Regression Analyses to Identify Factors Associated with LTL (Table 3)}

The univariate and multivariable regression analysis showed that LTL was inversely correlated with BMI, WC, HC, W-HR, W-htR, subscapular, lateral thoracic, $\Sigma 6 \mathrm{SF}$, subscapular/triceps ratio, and central skinfolds. Further, R-squared $\left(R^{2}\right)$ statistic for multivariable linear model after adjusted for age, family income, education and hypertension $L T L$ was significantly related to $B M I\left(R^{2}=0.91\right)$, WC $\left(R^{2}=0.83\right), H C\left(R^{2}=\right.$ $0.78)$, W-HR $\left(R^{2}=0.80\right), W-h t R\left(R^{2}=0.72\right)$, subscapular $\left(R^{2}=0.66\right)$, lateral thoracic $\left(R^{2}=0.80\right)$, central $\left(R^{2}\right.$ $=0.73)$, and $\Sigma 6 S F,\left(R^{2}=0.75\right)$. 
Table 3

Univariate and multivariable linear regression analyses to identify independent effect of leucocyte telomere length on obesity measures

\begin{tabular}{|c|c|c|c|c|c|}
\hline Parameters & $\begin{array}{l}\text { Un-adjusted regression } \\
\text { coefficient }(95 \% \mathrm{Cl})\end{array}$ & $\begin{array}{l}P \\
\text { value }\end{array}$ & $\begin{array}{l}\text { Adjusted } \\
\text { regression coefficient } \\
(95 \% \mathrm{Cl})\end{array}$ & $\begin{array}{l}P \\
\text { value }\end{array}$ & $\mathbf{R}^{2}$ \\
\hline $\begin{array}{l}\mathrm{BMI} \\
\left(\mathrm{Kg} / \mathrm{m}^{2}\right)\end{array}$ & $-109.7(-109.4,0.98 .1)$ & $<.0001$ & $-108.4(-106.2,-98.5)$ & $<.0001$ & 0.91 \\
\hline WC (cm) & $-135.4(-139.7,-131.0)$ & $<.0001$ & $\begin{array}{l}-134.17(-138.69, \\
-129.6)\end{array}$ & $<.0001$ & 0.83 \\
\hline $\mathrm{HC}(\mathrm{cm})$ & $-138.9(-144.0,-133.7)$ & $<.0001$ & $\begin{array}{l}-137.12(-142.4, \\
-131.8)\end{array}$ & $<.0001$ & 0.78 \\
\hline W-HR & $-0.05(-0.07,-0.03)$ & $<.0001$ & $-0.05(-0.08-0.03)$ & $<.0001$ & 0.80 \\
\hline W-htR & $-0.94(-0.98,-0.90)$ & $\begin{array}{l}< \\
0.0001\end{array}$ & $-0.93(-0.97,-0.88)$ & $\begin{array}{l}<.0001 \\
0.000\end{array}$ & 0.72 \\
\hline \multicolumn{6}{|c|}{ Skinfolds $(\mathrm{mm})$ and ratios } \\
\hline Biceps & $-0.89(-0.75,-0.98)$ & 0.81 & $-1.80(-1.76,-1.98)$ & 0.70 & 0.001 \\
\hline Triceps & $-0.80(-0.76,-0.92)$ & 0.94 & $-1.89(-1.68,-1.76)$ & 0.81 & 0.005 \\
\hline $\begin{array}{l}\text { Anterior } \\
\text { axillary }\end{array}$ & $-112.4(-91.5,-121.3)$ & $<.0001$ & $-115.3(-98.3,-120.1)$ & $\begin{array}{l}< \\
0.0001\end{array}$ & 0.42 \\
\hline Subscapular & $-157.4(-165.5,-149.3)$ & $<.0001$ & $-156.4(-164.7,-148.1)$ & $<.0001$ & 0.66 \\
\hline $\begin{array}{l}\text { Lateral } \\
\text { thoracic }\end{array}$ & $-200.1(-207.2,-193.0)$ & $\begin{array}{l}<.0001 \\
0.00\end{array}$ & $-198.8(-206.1,-191.5)$ & $\begin{array}{l}<.0001 \\
0.00\end{array}$ & 0.80 \\
\hline Suprailiac & $-200.7(-208.3,-193.1)$ & $<.0001$ & $-199.4(-207.2,-191.6)$ & $<.0001$ & 0.78 \\
\hline $\begin{array}{l}\text { Subscapular } \\
\text { /triceps } \\
\text { ratio }\end{array}$ & $-1.05(-1.20,-0.90)$ & $<.0001$ & $-1.07(-1.22,-0.92)$ & $<.0001$ & 0.22 \\
\hline Central & $-358.1(-373.8,-342.5)$ & $<.0001$ & $-355.9(-372.0,-339.8)$ & $\begin{array}{l}< \\
0.0001\end{array}$ & 0.73 \\
\hline Peripheral & $-0.85(-17.7,16.0)$ & 0.92 & $-1.89(-19.6,15.84)$ & 0.83 & 0.001 \\
\hline 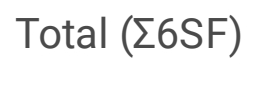 & $-760.6(-791.3,-730.0)$ & $\begin{array}{l}< \\
0.0001\end{array}$ & $\begin{array}{l}-755.07(-786.7 \\
-723.43)\end{array}$ & $\begin{array}{l}< \\
0.0001\end{array}$ & 0.75 \\
\hline
\end{tabular}




\section{Relationship between Obesity Measures and LTL According to LTL Quartiles (Table 5, Fig. 5)}

The association of measures of obesity and LTL revealed a significant negative correlation by multivariable linear regression analyses adjusted for age, family income, education, and hypertension, using the quartile 1 as reference. Multivariable linear regression analysis identified $B M I\left(r^{2}=0.93, p<\right.$ $0.0001)$, central skinfolds $\left(R^{2}=0.92, p<0.0001\right)$ and $\Sigma 6 S F,\left(R^{2}=0.90, p<0.0001\right)$ are independent predictors of LTL. 
Table 4

Demographic and clinical profiles, body composition and biochemical investigations of the subjects according to quartile of leukocyte telomeres length.

\begin{tabular}{|c|c|c|c|c|c|c|}
\hline Variables & $\begin{array}{l}\text { Quartile I } \\
\text { (LTL ratio < } \\
0.83)\end{array}$ & $\begin{array}{l}\text { Quartile } 2 \\
\text { (LTL ratio< } \\
0.87)\end{array}$ & $\begin{array}{l}\text { Quartile } 3 \\
\text { (LTL ratio< } \\
0.93)\end{array}$ & $\begin{array}{l}\text { Quartile } 4 \\
(\text { LTL ratio < } \\
0.98)\end{array}$ & $\begin{array}{l}\text { Between } \\
\text { groups } \\
\text { p-value }\end{array}$ & $\begin{array}{l}\text { Trend } p \text { - } \\
\text { value }\end{array}$ \\
\hline $\begin{array}{l}\text { Numbers } \\
(\%)\end{array}$ & $218(27.35)$ & $211(26.47)$ & $182(22.84)$ & $186(23.34)$ & $<0.0001$ & $<0.0001$ \\
\hline Age (Years) & $\begin{array}{l}45.71 \pm \\
9.52\end{array}$ & $\begin{array}{l}43.78 \pm \\
10.36\end{array}$ & $\begin{array}{l}40.32 \pm \\
9.76\end{array}$ & $\begin{array}{l}37.37 \pm \\
13.85\end{array}$ & $<0.00001$ & $<.00001$ \\
\hline \multicolumn{7}{|c|}{ Blood pressure (mmHg) } \\
\hline Systolic & $\begin{array}{l}127.20 \pm \\
11.87\end{array}$ & $\begin{array}{l}124.11 \pm \\
11.49\end{array}$ & $\begin{array}{l}120.21 \pm \\
13.23\end{array}$ & $\begin{array}{l}117.96 \pm \\
12.61\end{array}$ & $<0.0001$ & $<0.0001$ \\
\hline Diastolic & $\begin{array}{l}84.20 \pm \\
7.55\end{array}$ & $\begin{array}{l}81.56 \pm \\
6.80\end{array}$ & $\begin{array}{l}78.90 \pm \\
7.33\end{array}$ & $\begin{array}{l}76.66 \pm \\
7.91\end{array}$ & $<0.0001$ & $<0.0001$ \\
\hline \multicolumn{7}{|c|}{ Body composition } \\
\hline Weight (Kg) & $\begin{array}{l}74.52 \pm \\
9.09\end{array}$ & $\begin{array}{l}64.46 \pm \\
5.07\end{array}$ & $\begin{array}{l}58.44 \pm \\
4.68\end{array}$ & $\begin{array}{l}47.43 \pm \\
7.15\end{array}$ & $<0.0001$ & $<0.0001$ \\
\hline Height (cm) & $\begin{array}{l}152.21 \pm \\
5.90\end{array}$ & $\begin{array}{l}153.0 \pm \\
5.39\end{array}$ & $\begin{array}{l}154.70 \pm \\
5.92\end{array}$ & $\begin{array}{l}154.31 \pm \\
6.14\end{array}$ & $<0.0001$ & $<0.0001$ \\
\hline $\mathrm{BMI}\left(\mathrm{Kg} / \mathrm{m}^{2}\right)$ & $\begin{array}{l}32.44 \pm \\
3.89\end{array}$ & $\begin{array}{l}27.50 \pm \\
0.83\end{array}$ & $\begin{array}{l}24.40 \pm \\
0.86\end{array}$ & $\begin{array}{l}19.84 \pm \\
2.36\end{array}$ & $<0.0001$ & $<0.0001$ \\
\hline \multicolumn{7}{|c|}{$\begin{array}{l}\text { Circumferences }(\mathrm{cm}) \text { and } \\
\text { ratios }\end{array}$} \\
\hline Waist & $\begin{array}{l}97.69 \pm \\
4.12\end{array}$ & $\begin{array}{l}90.98 \pm \\
0.52\end{array}$ & $\begin{array}{l}88.29 \pm \\
2.08\end{array}$ & $\begin{array}{l}78.05 \pm \\
2.28\end{array}$ & $<0.00001$ & $<.00001$ \\
\hline Hip & $\begin{array}{l}104.54 \pm \\
4.34\end{array}$ & $\begin{array}{l}98.24 \pm \\
0.43\end{array}$ & $\begin{array}{l}96.36 \pm \\
1.70\end{array}$ & $\begin{array}{l}84.55 \pm \\
4.79\end{array}$ & $<0.00001$ & $<.00001$ \\
\hline W-HR & $\begin{array}{l}0.93 \pm \\
0.009\end{array}$ & $\begin{array}{l}0.92 \pm \\
0.004\end{array}$ & $\begin{array}{l}0.91 \pm \\
0.007\end{array}$ & $0.90 \pm 0.02$ & $<0.00001$ & $\begin{array}{l}< \\
0.00001\end{array}$ \\
\hline W-htR & $0.64 \pm 0.03$ & $0.59 \pm 0.02$ & $0.57 \pm 0.02$ & $0.50 \pm 0.02$ & $<0.00001$ & $<.00001$ \\
\hline \multicolumn{7}{|c|}{ Skinfolds $(\mathrm{mm})$ and ratios } \\
\hline Biceps & $\begin{array}{l}34.98 \pm \\
4.44\end{array}$ & $\begin{array}{l}27.05 \pm \\
0.49\end{array}$ & $\begin{array}{l}25.41 \pm \\
0.49\end{array}$ & $\begin{array}{l}19.54 \pm \\
3.12\end{array}$ & $<0.00001$ & $\begin{array}{l}< \\
0.00001\end{array}$ \\
\hline
\end{tabular}

Values are given as the mean \pm standard deviation and percentage (\%). BMI, body mass index; W-HR, waist-hip ratio; W-htR, waist-height ratio; CSF, central skinfold; FBG, fasting blood glucose. $P$ value is < 0.05 is statistically significant. 


\begin{tabular}{|c|c|c|c|c|c|c|}
\hline Variables & $\begin{array}{l}\text { Quartile I } \\
\text { (LTL ratio< } \\
0.83 \text { ) }\end{array}$ & $\begin{array}{l}\text { Quartile } 2 \\
\text { (LTL ratio < } \\
0.87 \text { ) }\end{array}$ & $\begin{array}{l}\text { Quartile } 3 \\
\text { (LTL ratio < } \\
0.93 \text { ) }\end{array}$ & $\begin{array}{l}\text { Quartile } 4 \\
\text { (LTL ratio< } \\
0.98)\end{array}$ & $\begin{array}{l}\text { Between } \\
\text { groups } \\
\text { p-value }\end{array}$ & $\begin{array}{l}\text { Trend } p \text { - } \\
\text { value }\end{array}$ \\
\hline Triceps & $\begin{array}{l}36.70 \pm \\
3.83\end{array}$ & $\begin{array}{l}28.34 \pm \\
0.56\end{array}$ & $\begin{array}{l}25.91 \pm \\
0.84\end{array}$ & $\begin{array}{l}22.17 \pm \\
2.17\end{array}$ & $<0.00001$ & $<.00001$ \\
\hline $\begin{array}{l}\text { Anterior } \\
\text { axillary }\end{array}$ & $\begin{array}{l}30.30 \pm \\
3.71\end{array}$ & $\begin{array}{l}26.31 \pm \\
0.71\end{array}$ & $\begin{array}{l}22.21 \pm \\
0.81\end{array}$ & $\begin{array}{l}18.17 \pm \\
2.13\end{array}$ & $<0.00001$ & $\begin{array}{l}<.00001 \\
0.00\end{array}$ \\
\hline Subscapular & $\begin{array}{l}49.85 \pm \\
4.76\end{array}$ & $33.19 \pm 3.0$ & $\begin{array}{l}28.24 \pm \\
0.92\end{array}$ & $\begin{array}{l}26.45 \pm \\
0.64\end{array}$ & $<0.00001$ & $<.00001$ \\
\hline $\begin{array}{l}\text { Lateral } \\
\text { thoracic }\end{array}$ & $52.59 \pm 4.4$ & $\begin{array}{l}36.68 \pm \\
3.68\end{array}$ & $\begin{array}{l}29.40 \pm \\
1.02\end{array}$ & $\begin{array}{l}23.99 \pm \\
1.74\end{array}$ & $<0.00001$ & $\begin{array}{l}<.00001 \\
0.0\end{array}$ \\
\hline Suprailiac & $\begin{array}{l}54.40 \pm \\
4.63\end{array}$ & $37.37 \pm 3.3$ & $\begin{array}{l}30.72 \pm \\
1.38\end{array}$ & $\begin{array}{l}25.22 \pm \\
1.06\end{array}$ & $<0.00001$ & $\begin{array}{l}<.00001 \\
0.0\end{array}$ \\
\hline $\begin{array}{l}\text { Subscapular } \\
\text { /triceps ratio }\end{array}$ & $1.25 \pm 0.02$ & $1.16 \pm 0.08$ & $1.09 \pm 0.18$ & $1.04 \pm 0.09$ & $<0.00001$ & $\begin{array}{l}<.00001 \\
0.0\end{array}$ \\
\hline Central & $\begin{array}{l}104.26 \pm \\
9.3\end{array}$ & $\begin{array}{l}70.57 \pm \\
6.24\end{array}$ & $\begin{array}{l}58.97 \pm \\
2.24\end{array}$ & $\begin{array}{l}51.68 \pm \\
1.55\end{array}$ & $<0.00001$ & $<.00001$ \\
\hline Peripheral & $\begin{array}{l}56.63 \pm \\
12.52\end{array}$ & $\begin{array}{l}55.85 \pm \\
11.5\end{array}$ & $\begin{array}{l}56.03 \pm \\
11.03\end{array}$ & $\begin{array}{l}56.53 \pm \\
13.1\end{array}$ & 0.89 & 0.597 \\
\hline 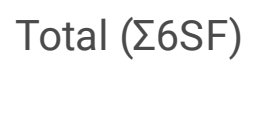 & $\begin{array}{l}258.82 \pm \\
25.81\end{array}$ & $\begin{array}{l}188.94 \pm \\
11.74\end{array}$ & $\begin{array}{l}161.89 \pm \\
5.46\end{array}$ & $\begin{array}{l}135.54 \pm \\
10.86\end{array}$ & $<0.00001$ & $<.00001$ \\
\hline \multicolumn{7}{|c|}{ Biochemical Investigations } \\
\hline $\begin{array}{l}\text { FBG } \\
(\mathrm{mg} / \mathrm{dL})\end{array}$ & $\begin{array}{l}113.50 \pm \\
8.20\end{array}$ & $\begin{array}{l}111.01 \pm \\
7.20\end{array}$ & $105 \pm 8.40$ & $\begin{array}{l}101.59 \pm \\
8.04\end{array}$ & $<0.00001$ & $\begin{array}{l}<.00001 \\
0.00\end{array}$ \\
\hline $\begin{array}{l}\text { Vitamin D } \\
(\mathrm{ng} / \mathrm{ml})\end{array}$ & $\begin{array}{l}19.84 \pm \\
2.36\end{array}$ & $\begin{array}{l}24.44 \pm \\
0.86\end{array}$ & $\begin{array}{l}27.50 \pm \\
0.83\end{array}$ & $\begin{array}{l}32.44 \pm \\
3.89\end{array}$ & $<0.0001$ & $<0.0001$ \\
\hline
\end{tabular}


Table 5

Univariate and multivariable linear regression analyses to identify independent predictors of leukocyte telomere length (LTL) according to LTL quartiles.

\begin{tabular}{|c|c|c|c|c|c|c|}
\hline Parameters & Quartile & $\begin{array}{l}\text { Un-adjusted } \\
\text { regression } \\
\text { coefficient }\end{array}$ & $p$ & $\begin{array}{l}\text { Adjusted } \\
\text { regression } \\
\text { coefficient }\end{array}$ & p & $\mathrm{R}^{2}$ \\
\hline \multirow{4}{*}{$\begin{array}{l}\text { Body mass index } \\
\left(\mathrm{Kg} / \mathrm{m}^{2}\right)\end{array}$} & Quartile1 & - & & - & & \multirow[t]{4}{*}{0.93} \\
\hline & $\begin{array}{l}\text { Quartile } \\
2\end{array}$ & $\begin{array}{l}-16.4(-16.5 \\
-17.3)\end{array}$ & $\begin{array}{l}<.0001 \\
0.00\end{array}$ & $\begin{array}{l}-16.9(-17.5 \\
-16.3)\end{array}$ & $\begin{array}{l}<.0001 \\
0.0\end{array}$ & \\
\hline & $\begin{array}{l}\text { Quartile } \\
3\end{array}$ & $\begin{array}{l}-22.5(-25.1 \\
-21.9)\end{array}$ & $\begin{array}{l}< \\
0.0001\end{array}$ & $\begin{array}{l}-23.5(-24.1 \\
-22.9)\end{array}$ & $\begin{array}{l}< \\
0.0001\end{array}$ & \\
\hline & $\begin{array}{l}\text { Quartile } \\
4\end{array}$ & $\begin{array}{l}-29.7(-28.7 \\
-28.4)\end{array}$ & $\begin{array}{l}<.0001 \\
0.00\end{array}$ & $\begin{array}{l}-29.0(-29.7 \\
-28.4)\end{array}$ & $\begin{array}{l}<.0001 \\
0.0\end{array}$ & \\
\hline \multirow[t]{4}{*}{$\begin{array}{l}\text { Waist } \\
\text { circumference }(\mathrm{cm})\end{array}$} & $\begin{array}{l}\text { Quartile } \\
1\end{array}$ & - & & - & & \multirow[t]{4}{*}{0.87} \\
\hline & $\begin{array}{l}\text { Quartile } \\
2\end{array}$ & $\begin{array}{l}-6.71(-7.21, \\
-6.21)\end{array}$ & $\begin{array}{l}<.0001 \\
0.00\end{array}$ & $-6.6(-7.1,-6.1)$ & $\begin{array}{l}<.0001 \\
0.0\end{array}$ & \\
\hline & $\begin{array}{l}\text { Quartile } \\
3\end{array}$ & $\begin{array}{l}-9.40(-9.92 \\
-8.82)\end{array}$ & $\begin{array}{l}< \\
0.0001\end{array}$ & $\begin{array}{l}-9.30(-9.8 \\
-8.70)\end{array}$ & $\begin{array}{l}<.0001 \\
0.00\end{array}$ & \\
\hline & $\begin{array}{l}\text { Quartile } \\
4\end{array}$ & $\begin{array}{l}-19.63(-20.13 \\
-19.12)\end{array}$ & $\begin{array}{l}< \\
0.0001\end{array}$ & $\begin{array}{l}-19.5(-20.6, \\
-18.9)\end{array}$ & $\begin{array}{l}< \\
0.0001\end{array}$ & \\
\hline \multirow[t]{4}{*}{$\begin{array}{l}\text { Hip circumference } \\
\text { (cm) }\end{array}$} & $\begin{array}{l}\text { Quartile } \\
1\end{array}$ & - & & - & & \multirow[t]{4}{*}{0.82} \\
\hline & $\begin{array}{l}\text { Quartile } \\
2\end{array}$ & $\begin{array}{l}-6.29(-6.91 \\
-0.566)\end{array}$ & $\begin{array}{l}<.0001 \\
0.000\end{array}$ & $-6.2(-6.9,-5.6)$ & $\begin{array}{l}<.0001 \\
0.00\end{array}$ & \\
\hline & $\begin{array}{l}\text { Quartile } \\
3\end{array}$ & $\begin{array}{l}-8.17(-8.82 \\
-7.51)\end{array}$ & $\begin{array}{l}<.0001 \\
0.000\end{array}$ & $\begin{array}{l}-8.05(-8.7 \\
-7.3)\end{array}$ & $\begin{array}{l}< \\
0.0001\end{array}$ & \\
\hline & $\begin{array}{l}\text { Quartile } \\
4\end{array}$ & $\begin{array}{l}-19.98(-20.64, \\
-19.33)\end{array}$ & $\begin{array}{l}<.0001 \\
0.00\end{array}$ & $\begin{array}{l}-19.7(-20.4 \\
-19.0)\end{array}$ & $\begin{array}{l}<.0001 \\
0.0\end{array}$ & \\
\hline \multirow[t]{4}{*}{ Waist-hip ratio } & $\begin{array}{l}\text { Quartile } \\
1\end{array}$ & - & & - & & \multirow[t]{4}{*}{0.84} \\
\hline & $\begin{array}{l}\text { Quartile } \\
2\end{array}$ & $\begin{array}{l}-0.008(-0.011 \\
-0.005)\end{array}$ & $\begin{array}{l}<.0001 \\
0.00\end{array}$ & $\begin{array}{l}-0.008(-0.01- \\
0.005)\end{array}$ & $\begin{array}{l}<.0001 \\
0.0\end{array}$ & \\
\hline & $\begin{array}{l}\text { Quartile } \\
3\end{array}$ & $\begin{array}{l}-0.018(-0.021, \\
0.015)\end{array}$ & $\begin{array}{l}< \\
0.0001\end{array}$ & $\begin{array}{l}-0.18(-0.21, \\
-0.15\end{array}$ & $\begin{array}{l}< \\
0.0001\end{array}$ & \\
\hline & $\begin{array}{l}\text { Quartile } \\
4\end{array}$ & $\begin{array}{l}-0.009(-0.012 \\
-0.006)\end{array}$ & $\begin{array}{l}< \\
0.0001\end{array}$ & $\begin{array}{l}-0.01(-0.01 \\
-0.007)\end{array}$ & $\begin{array}{l}< \\
0.0001\end{array}$ & \\
\hline
\end{tabular}

Adjusted for age, family income, education, and hypertension. $P$ value is $<0.05$ is statistically significant. 


\begin{tabular}{|c|c|c|c|c|c|c|}
\hline Parameters & Quartile & $\begin{array}{l}\text { Un-adjusted } \\
\text { regression } \\
\text { coefficient }\end{array}$ & p & $\begin{array}{l}\text { Adjusted } \\
\text { regression } \\
\text { coefficient }\end{array}$ & $\mathbf{p}$ & $\mathrm{R}^{2}$ \\
\hline \multirow[t]{4}{*}{ Waist-height ratio } & $\begin{array}{l}\text { Quartile } \\
1\end{array}$ & - & & - & & \multirow[t]{4}{*}{0.74} \\
\hline & $\begin{array}{l}\text { Quartile } \\
2\end{array}$ & $\begin{array}{l}-0.047(-0.053 \\
-0.042)\end{array}$ & $\begin{array}{l}< \\
0.0001\end{array}$ & $\begin{array}{l}-0.04(-0.05 \\
-0.04)\end{array}$ & $\begin{array}{l}< \\
0.0001\end{array}$ & \\
\hline & $\begin{array}{l}\text { Quartile } \\
3\end{array}$ & $\begin{array}{l}-0.07(-0.07 \\
-0.06)\end{array}$ & $\begin{array}{l}< \\
0.0001\end{array}$ & $\begin{array}{l}-0.07(-0.07, \\
-0.06)\end{array}$ & $\begin{array}{l}< \\
0.0001\end{array}$ & \\
\hline & $\begin{array}{l}\text { Quartile } \\
4\end{array}$ & $\begin{array}{l}-0.132(-0.141 \\
-0.130)\end{array}$ & <. 0001 & $\begin{array}{l}-0.13(-0.14, \\
-0.12)\end{array}$ & $\begin{array}{l}<.0001 \\
0.0\end{array}$ & \\
\hline \multirow{4}{*}{$\begin{array}{l}\text { Subscapular } \\
\text { /triceps ratio }\end{array}$} & $\begin{array}{l}\text { Quartile } \\
1\end{array}$ & - & & - & & \multirow[t]{4}{*}{0.69} \\
\hline & $\begin{array}{l}\text { Quartile } \\
2\end{array}$ & $\begin{array}{l}-0.18(-0.20 \\
-0.17)\end{array}$ & $\begin{array}{l}<.0001 \\
0.000\end{array}$ & $\begin{array}{l}-0.18(-0.20, \\
-0.17)\end{array}$ & $\begin{array}{l}<.0001 \\
\vdots\end{array}$ & \\
\hline & $\begin{array}{l}\text { Quartile } \\
3\end{array}$ & $\begin{array}{l}-0.26(-0.28 \\
-0.25)\end{array}$ & $\begin{array}{l}<.0001 \\
0.00\end{array}$ & $\begin{array}{l}-0.26(-0.28, \\
-0.25)\end{array}$ & $\begin{array}{l}<.0001 \\
0.00\end{array}$ & \\
\hline & $\begin{array}{l}\text { Quartile } \\
4\end{array}$ & $\begin{array}{l}-0.15(-0.170 \\
-0.14)\end{array}$ & $\begin{array}{l}<.0001 \\
0.00\end{array}$ & $\begin{array}{l}-0.16(-0.17, \\
-0.14)\end{array}$ & $\begin{array}{l}<.0001 \\
0.00\end{array}$ & \\
\hline \multirow[t]{4}{*}{ Central skinfolds } & $\begin{array}{l}\text { Quartile } \\
1\end{array}$ & - & & - & & \multirow[t]{4}{*}{0.92} \\
\hline & $\begin{array}{l}\text { Quartile } \\
2\end{array}$ & $\begin{array}{l}-33.6(-34.82 \\
-32.5)\end{array}$ & $\begin{array}{l}< \\
0.0001\end{array}$ & $\begin{array}{l}-33.5(-34.7 \\
-32.4)\end{array}$ & $\begin{array}{l}< \\
0.0001\end{array}$ & \\
\hline & $\begin{array}{l}\text { Quartile } \\
3\end{array}$ & $\begin{array}{l}-45.29(-46.47 \\
-44.11)\end{array}$ & $\begin{array}{l}<.0001 \\
0.00\end{array}$ & $\begin{array}{l}-45.0(-46.2, \\
-43.8)\end{array}$ & $\begin{array}{l}<.0001 \\
0.00\end{array}$ & \\
\hline & $\begin{array}{l}\text { Quartile } \\
4\end{array}$ & $\begin{array}{l}-52.5(-53.7 \\
-51.40)\end{array}$ & $\begin{array}{l}<.0001 \\
0.000\end{array}$ & $\begin{array}{l}-52.3(-53.8, \\
-51.1\end{array}$ & $\begin{array}{l}< \\
0.0001\end{array}$ & \\
\hline \multirow[t]{4}{*}{$\begin{array}{l}\text { Peripheral } \\
\text { skinfolds }\end{array}$} & $\begin{array}{l}\text { Quartile } \\
1\end{array}$ & - & & - & & \multirow[t]{5}{*}{0.0008} \\
\hline & $\begin{array}{l}\text { Quartile } \\
2\end{array}$ & $\begin{array}{l}-0.77(-3.07 \\
1.51)\end{array}$ & 0.51 & - & & \\
\hline & $\begin{array}{l}\text { Quartile } \\
3\end{array}$ & $\begin{array}{l}-0.59(-2.98, \\
1.78)\end{array}$ & 0.62 & - & & \\
\hline & $\begin{array}{l}\text { Quartile } \\
4\end{array}$ & $\begin{array}{l}-0.098(-2.48 \\
2.29)\end{array}$ & 0.93 & - & & \\
\hline $\begin{array}{l}\text { Total skinfolds } \\
(\Sigma 6 \mathrm{SF})\end{array}$ & $\begin{array}{l}\text { Quartile } \\
1\end{array}$ & - & & - & & \\
\hline
\end{tabular}




\begin{tabular}{|c|c|c|c|c|c|c|}
\hline Parameters & Quartile & $\begin{array}{l}\text { Un-adjusted } \\
\text { regression } \\
\text { coefficient }\end{array}$ & $p$ & $\begin{array}{l}\text { Adjusted } \\
\text { regression } \\
\text { coefficient }\end{array}$ & $p$ & $\mathrm{R}^{2}$ \\
\hline & $\begin{array}{l}\text { Quartile } \\
2\end{array}$ & $\begin{array}{l}-65.88(-68.55 \\
-63.21)\end{array}$ & $\begin{array}{l}<.0001 \\
0.000\end{array}$ & $\begin{array}{l}-65.6(-68.3 \\
-62.9)\end{array}$ & $\begin{array}{l}<.0001 \\
0.0\end{array}$ & \multirow[t]{3}{*}{0.90} \\
\hline & $\begin{array}{l}\text { Quartile } \\
3\end{array}$ & $\begin{array}{l}-88.25(-91.03 \\
-85.48)\end{array}$ & $\begin{array}{l}< \\
0.0001\end{array}$ & $\begin{array}{l}-87.6(-90.4, \\
-84.8)\end{array}$ & $\begin{array}{l}< \\
0.0001\end{array}$ & \\
\hline & $\begin{array}{l}\text { Quartile } \\
4\end{array}$ & $\begin{array}{l}-111.1(-113.9 \\
-108.40)\end{array}$ & $<.0001$ & $\begin{array}{l}-110.5(-113 \\
-107)\end{array}$ & $\begin{array}{l}< \\
0.0001\end{array}$ & \\
\hline
\end{tabular}

\section{Discussion}

This is the first study to investigate the relationship of LTL in women with prediabetes. Specifically, relationship of LTL with subcutaneous adiposity, particularly truncal adiposity is being reported for the first time.

The association between obesity and telomerase physiology has been investigated previously. Previously, some research studies have shown significant negative relationship between adiposity measures and LTL $(19,20)$, while others did not $(21,22)$. Associations of LTL with generalized and abdominal obesity, as well as weight changes was researched in China (n, 2,912 healthy Chinese women ages 40-70 years) (23). Interestingly, like our study, this study was done exclusively in women. This study showed that women who maintained their weight within $+/-5 \%$ since age 50 , or reduced their weight, had a longer mean of current telomerase length than women who gained weight since age 50(23). A study in white women ( $n, 1122$ aged 18-76 years) in UK showed that the telomerase of obese women were $240 \mathrm{bp}$ shorter than those of lean women (24). In a meta-analysis (87 observational studies including data from 146114 subjects), Gielen et al (25) showed a significant inverse relationship between BMI and LTL particularly in younger population without any gender differences. These associations were predominantly for the white pooled population (25).

Asian Indians have thicker subcutaneous adipose tissue than white Caucasians(26). Specifically, deep subcutaneous adipose tissue on posterior aspects in lumbar region may be of importance for insulin resistance (27). Overall, apart from intra-abdominal adipose tissue, subcutaneous adipose tissue in truncal and abdominal area are higher and are of greater metabolic importance to Asian Indians than other races and this adipose tissue mass was associated with other components of metabolic syndrome (e.g. non-alcoholic fatty liver diseases) $(28,29)$. Moreover, abdominal adiposity indicates high cardiovascular risk in this ethnic group (30). Only a few studies have shown relationship of multiple measures of obesity with LTL. Cui et al (23) showed inverse association between LTL and weight, WC, HC, BMI and W-htR, but not with W-HR or height in Chinese women. In non-Hispanic whites (Fels Longitudinal 
Study, 309 subjects aged 8 to 80 yrs., 52\% females) WC, HC, total body fat, and visceral adipose tissue volume were inversely associated with telomerase length at borderline significance (31). In current study both abdominal and truncal adiposity are related to $L T L$, the association of latter with LTL has not been investigated before.

Relationship between hyperglycemia and telomerase has been researched more often. A study in mice deficient for the telomerase RNA component gene shown that short LTL reduces $\beta$ cell mass and subsequent impaired insulin secretion and glucose tolerance(32). Human observational case control study from a community based white population (432 T2DM and 424 controls) have shown decreased log (e) transformed mean leukocyte telomerase repeat copy number to single gene copy number was significantly associated with T2DM (33). Recent study from north India (n, 1354 individuals 682 cases of T2DM and 672 healthy controls), genotyped for 12 Variants from 7 telomerase maintenance genes showed significant association of five variants with T2DM. (34) Zhou et al., (35) recruited 556 Chinese subjects [T2DM $(n, 159)$, pre-diabetes $(n, 197)$, and normal glucose tolerance $(n, 200)$ ] and suggested that shorter LTL was associated with higher $\mathrm{HbA} 1 \mathrm{c}$, fasting plasma glucose, postprandial glucose and lower glucose-stimulated insulin release. Interestingly these authors showed LTL shortening existed in individuals with pre-diabetes, and LTL was shortest in diabetes. Diabetes patients with better glycemic control $(\mathrm{HbA} 1 \mathrm{c}<7 \%)$ had longer LTL. Further, these authors also investigated relationship of LTL with diet taken by the subjects. They reported that legumes, nuts, fish and seaweeds were protective factors for LTL shortening, and sweetened carbonated beverage was a risk factor for LTL shortening(35). This was the only study, apart from present study, where individuals with prediabetes were investigated in context of LTL. An intervention study of pistachio intake in individuals with prediabetes has been briefly stated previously. This randomized clinical trial on forty-nine men and women (aged between 25-65 years, $\mathrm{BMI} \leq 35 \mathrm{~kg} / \mathrm{m}^{2}$ ) with prediabetes showed that chronic pistachio consumption reduced oxidative damage to DNA and increased the gene expression of some telomerase-associated genes (12). In addition, lessening oxidative damage to DNA and telomerase expression through diet may represent an interesting way to promote health span in humans.

Having stated that, association of LTL with hyperglycemia in humans continues to be investigated and debated. Specifically, subjects with prediabetes have been less examined in the context of LTL. These data also highlight importance of data of the present study; first except a insufficient, most studies have been completed in limited number of subjects. Second, no investigator has specifically focused on women with prediabetes. Third, association of LTL with skinfolds thickness (hence subcutaneous and truncal adiposity) in individuals with prediabetes has not been shown previously. This study has the following limitations. First, the study targeted of people of one ethnic origin and a single region, which prevents generalization to the entire population and race. Second, as a cross-sectional study, sequential correlation could not be verified. Third, we did not estimate biomarkers of oxidative stress and inflammation, so we could not examine potential relationship between them and LTL. Fourth, longitudinal and intervention studies are necessary to study interplay between LTL, and prediabetes. 
Overall, our report clearly shows that in Individuals with prediabetes, presence of obesity, and truncal obesity may accelerate ageing in Asian Indian women with prediabetes.

\section{Abbreviations}

Fasting blood glucose, FBG; 2-h post oral glucose tolerance test, OGTT; 25 hydroxy vitamin D, 25(OH)D); body mass index, BMl; impaired fasting glucose, IFG; type 2 diabetes mellitus, T2DM; normal glucose tolerance, NGT; hemoglobin, $\mathrm{HbA} 1 \mathrm{c}$; total cholesterol, TC; serum triglycerides, TG; high-density lipoprotein cholesterol, HDL-c; low-density lipoprotein cholesterol, LDL-c.

\section{Declarations}

Acknowledgements: The cooperation of the subjects who took part in the study is greatly appreciated.

Funding: This research was supported from the Department of Science and Technology, Ministry of Science and Technology, Government of India (File Number: SSD/WS/098/2010). The funding agency had no role in study design, data collection, and analysis, decision to publish, or preparation of the manuscript.

Availability of data and materials: All data generated or analysed during this study are included in this article.

Authors Contributors: AM, conceived the study and contributed to the discussion and reviewed the manuscript. SPB, conducted the study, performed laboratory investigations and wrote the manuscript. RMP and ADU analysed and interpreted the data; AM is the guarantor for this manuscript.

Ethics Approval: Institutional ethics committee of Fortis C-DOC Centre of Excellence for Diabetes, Metabolic Diseases and Endocrinology, Chirag Enclave, Nehru Place, New Delhi..

Conflict of interest: None of the authors have any conflicts to disclose.

\section{References}

1. Misra A, Gopalan H, Jayawardena R, Hills AP, Soares M, Reza-Albarran AA, et al. Diabetes in developing countries. J Diabetes. 2019 Mar 12.

2. Misra A, Sattar N, Tandon N, Shrivastava U, Vikram NK, Khunti K, et al. Clinical management of type 2 diabetes in south Asia. Lancet Diabetes Endocrinol, . 2018;pii: S2213-8587(18)30199-2. doi: 10.1016/S2213-8587(18)30199-2.

3. Hills A, Misra A, Gill JM, Byrne N, Soares M, Ramachandran A, et al. Public health and health systems: implications for the prevention and management of type 2 diabetes in south Asia. Lancet Diabetes Endocrinol pii: S2213-8587(18)30203-1 doi: 101016/S2213-8587(18)30203-1. 2018. 
4. Clarke PM, Gray AM, Briggs A, Farmer AJ, Fenn P, Stevens RJ, et al. A model to estimate the lifetime health outcomes of patients with type 2 diabetes: the United Kingdom Prospective Diabetes Study (UKPDS) Outcomes Model (UKPDS no. 68). Diabetologia. 2004 Oct;47(10):1747-59.

5. Rao Kondapally Seshasai S, Kaptoge S, Thompson A, Di Angelantonio E, Gao P, Sarwar N, et al. Diabetes mellitus, fasting glucose, and risk of cause-specific death. N Engl J Med. 2011 Mar 3;364(9):829-41.

6. Muschik D, Tetzlaff J, Lange K, Epping J, Eberhard S, Geyer S. Change in life expectancy with type 2 diabetes: a study using claims data from lower Saxony, Germany. Popul Health Metr. 2017 Feb 13;15(1):5.

7. Houben JM, Moonen HJ, van Schooten FJ, Hageman GJ. Telomere length assessment: biomarker of chronic oxidative stress? Free Radic Biol Med. 2008 Feb 1;44(3):235-46.

8. Hornsby PJ. Telomerase and the aging process. Exp Gerontol. 2007 Jul;42(7):575-81.

9. Boccardi V, Paolisso G. Telomerase activation: a potential key modulator for human healthspan and longevity. Ageing Res Rev. 2014 May;15:1-5.

10. Butt HZ, Atturu G, London NJ, Sayers RD, Bown MJ. Telomere length dynamics in vascular disease: a review. Eur J Vasc Endovasc Surg. 2010 Jul;40(1):17-26.

11. Boccardi V, Esposito A, Rizzo MR, Marfella R, Barbieri M, Paolisso G. Mediterranean diet, telomere maintenance and health status among elderly. PLoS One. 2013;8(4):e62781.

12. Canudas S, Hernandez-Alonso P, Galie S, Muralidharan J, Morell-Azanza L, Zalba G, et al. Pistachio consumption modulates DNA oxidation and genes related to telomere maintenance: a crossover randomized clinical trial. Am J Clin Nutr. 2019 Jun 1;109(6):1738-45.

13. Bhatt SP, Misra A, Pandey RM, Upadhyay AD, Gulati S, Singh N. Vitamin D Supplementation in Overweight/obese Asian Indian Women with Prediabetes Reduces Glycemic Measures and Truncal Subcutaneous Fat: A 78 Weeks Randomized Placebo-Controlled Trial (PREVENT-WIN Trial). Sci Rep. 2020 Jan 14;10(1):220.

14. Bhatt SP, Misra A, Pandey RM. rs7903146 (C/T) polymorphism of Transcription factor 7 like 2 (TCF7L-2) gene is independently associated with non-alcoholic fatty liver disease in Asian Indians. Diabetes Metab Syndr. 2020 May - Jun;14(3):175-80.

15. Cawthon RM. Telomere measurement by quantitative PCR. Nucleic Acids Res. 2002 May 15;30(10):e47.

16. Lin J, Epel E, Cheon J, Kroenke C, Sinclair E, Bigos M, et al. Analyses and comparisons of telomerase activity and telomere length in human $T$ and $B$ cells: insights for epidemiology of telomere maintenance. J Immunol Methods. 2010 Jan 31;352(1-2):71-80.

17. Misra A, Chowbey P, Makkar BM, Vikram NK, Wasir JS, Chadha D, et al. Consensus statement for diagnosis of obesity, abdominal obesity and the metabolic syndrome for Asian Indians and recommendations for physical activity, medical and surgical management. J Assoc Physicians India. 2009 Feb;57:163-70.

18. Holick MF. Vitamin D deficiency. N Engl J Med. 2007 Jul 19;357(3):266-81. 
19. Fitzpatrick AL, Kronmal RA, Gardner JP, Psaty BM, Jenny NS, Tracy RP, et al. Leukocyte telomere length and cardiovascular disease in the cardiovascular health study. Am J Epidemiol. 2007 Jan 1;165(1):14-21.

20. Nordfjall K, Eliasson M, Stegmayr B, Melander O, Nilsson P, Roos G. Telomere length is associated with obesity parameters but with a gender difference. Obesity (Silver Spring). 2008 Dec;16(12):26829.

21. Bekaert S, De Meyer T, Rietzschel ER, De Buyzere ML, De Bacquer D, Langlois M, et al. Telomere length and cardiovascular risk factors in a middle-aged population free of overt cardiovascular disease. Aging Cell. 2007 Oct;6(5):639-47.

22. Nettleton JA, Diez-Roux A, Jenny NS, Fitzpatrick AL, Jacobs DR, Jr. Dietary patterns, food groups, and telomere length in the Multi-Ethnic Study of Atherosclerosis (MESA). Am J Clin Nutr. 2008 Nov;88(5):1405-12.

23. Cui Y, Gao YT, Cai Q, Qu S, Cai H, Li HL, et al. Associations of leukocyte telomere length with body anthropometric indices and weight change in Chinese women. Obesity (Silver Spring). 2013 Dec;21(12):2582-8.

24. Valdes AM, Andrew T, Gardner JP, Kimura M, Oelsner E, Cherkas LF, et al. Obesity, cigarette smoking, and telomere length in women. Lancet. 2005 Aug 20-26;366(9486):662-4.

25. Gielen M, Hageman GJ, Antoniou EE, Nordfjall K, Mangino M, Balasubramanyam M, et al. Body mass index is negatively associated with telomere length: a collaborative cross-sectional meta-analysis of 87 observational studies. Am J Clin Nutr. 2018 Sep 1;108(3):453-75.

26. Misra A, Jayawardena R, Anoop S. Obesity in South Asia: Phenotype, Morbidities, and Mitigation. Curr Obes Rep. 2019 Feb 7.

27. Misra A, Soares M, Mohan V, Anoop S, Abhishek V, Vaidy R, et al. Body Fat, Metabolic Syndrome and Hyperglycemia in South Asians. J Diabetes Complications (https://doiorg/101016/jjdiacomp201808001). 2018.

28. Bhatt SP, Misra A, Nigam P, Guleria R, Pasha MA. Phenotype, Body Composition, and Prediction Equations (Indian Fatty Liver Index) for Non-Alcoholic Fatty Liver Disease in Non-Diabetic Asian Indians: A Case-Control Study. PLoS One. 2015;10(11):e0142260.

29. Misra A, Soares MJ, Mohan V, Anoop S, Abhishek V, Vaidya R, et al. Body fat, metabolic syndrome and hyperglycemia in South Asians. J Diabetes Complications. 2018 Nov;32(11):1068-75.

30. Gulati S, Misra A. Abdominal obesity and type 2 diabetes in Asian Indians: dietary strategies including edible oils, cooking practices and sugar intake. Eur J Clin Nutr. 2017 Jul;71(7):850-7.

31. Lee M, Martin H, Firpo MA, Demerath EW. Inverse association between adiposity and telomere length: The Fels Longitudinal Study. Am J Hum Biol. 2011 Jan-Feb;23(1):100-6.

32. Kuhlow D, Florian S, von Figura G, Weimer S, Schulz N, Petzke KJ, et al. Telomerase deficiency impairs glucose metabolism and insulin secretion. Aging (Albany NY). 2010 Oct;2(10):650-8.

33. Zee RY, Castonguay AJ, Barton NS, Germer S, Martin M. Mean leukocyte telomere length shortening and type 2 diabetes mellitus: a case-control study. Transl Res. 2010 Apr;155(4):166-9. 
34. Sethi I, Sharma V, Sharma I, Singh G, Bhat GR, Bhanwer AJS, et al. Telomere Maintenance Genes are associated with Type 2 Diabetes Susceptibility in Northwest Indian Population Group. Sci Rep. 2020 Apr 15;10(1):6444.

35. Zhou M, Zhu L, Cui X, Feng L, Zhao X, He S, et al. Influence of diet on leukocyte telomere length, markers of inflammation and oxidative stress in individuals with varied glucose tolerance: a Chinese population study. Nutr J. 2016 Apr 12;15:39.

\section{Figures}




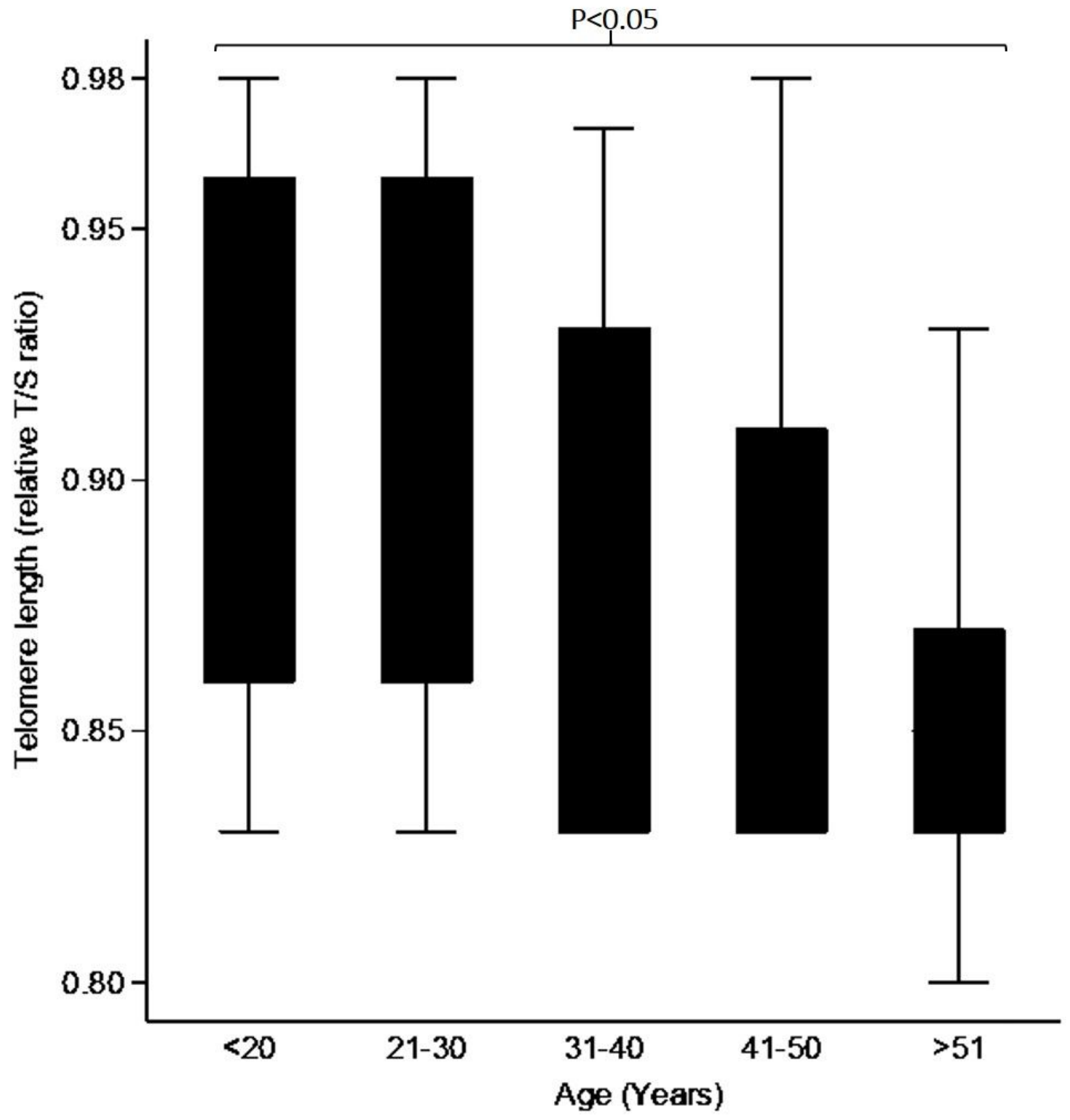

Figure 1

Figure 1

Leucocyte telomerase length according to age categories. 


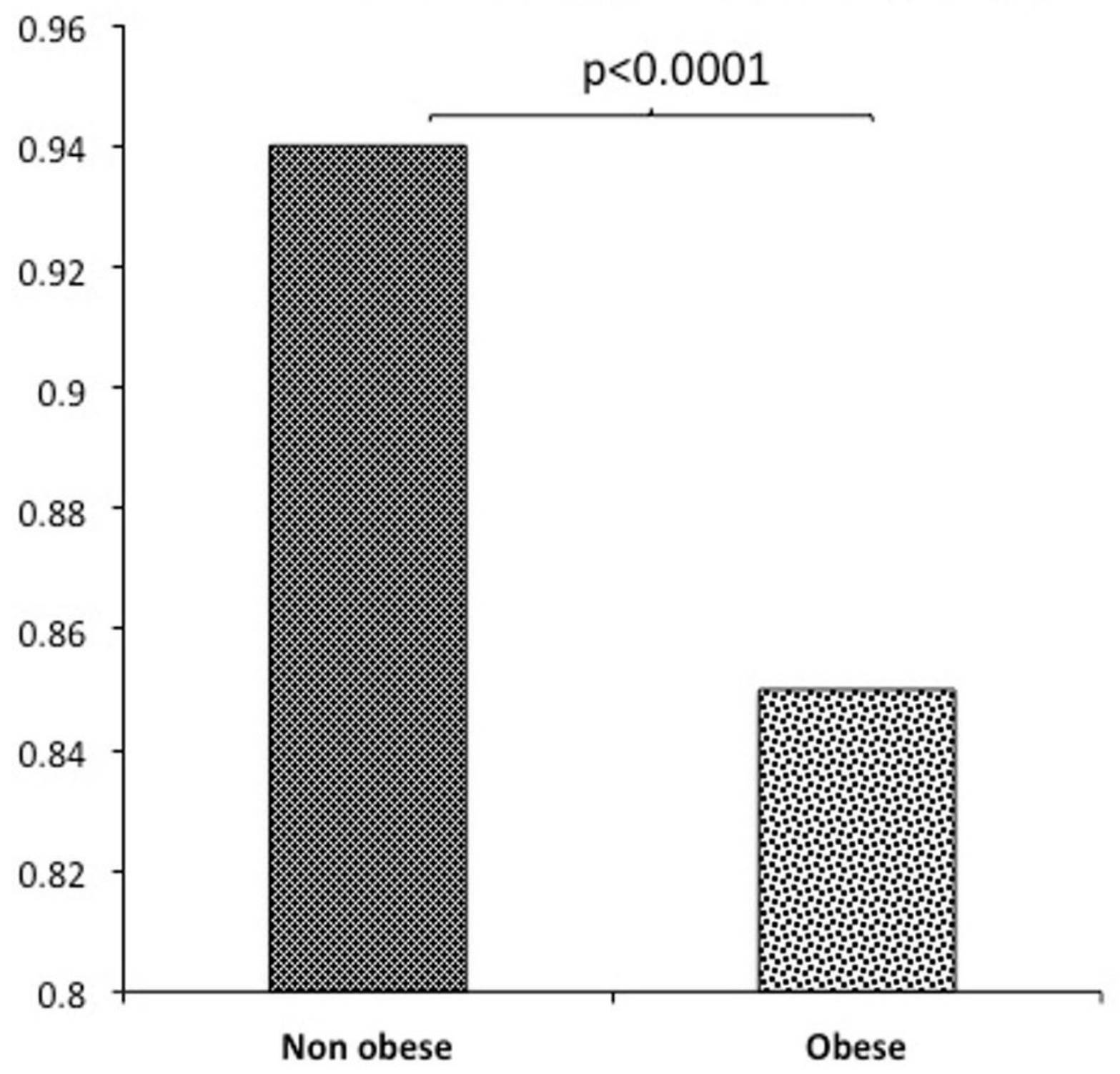

Figure 2

Figure 2

Leucocyte telomerase length in obese and non-obese women. 


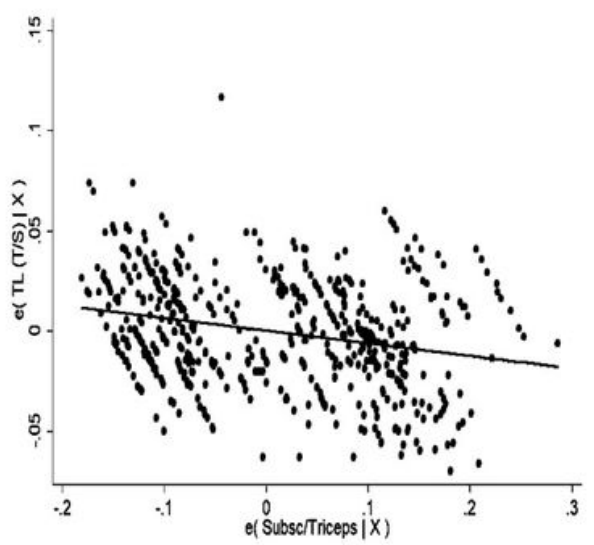

a

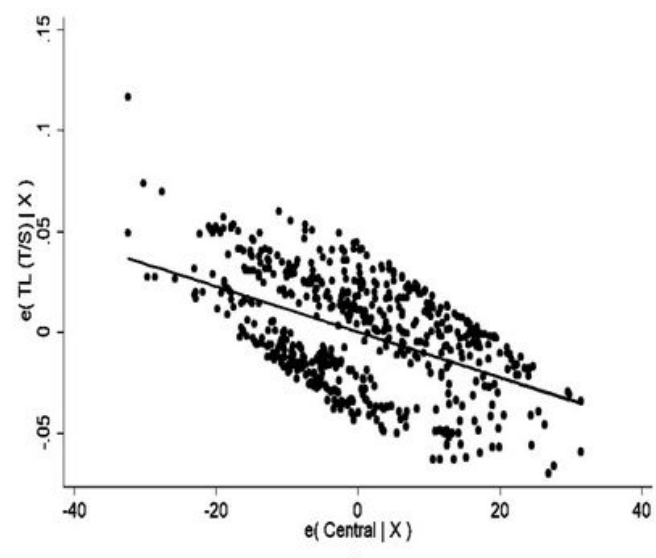

b

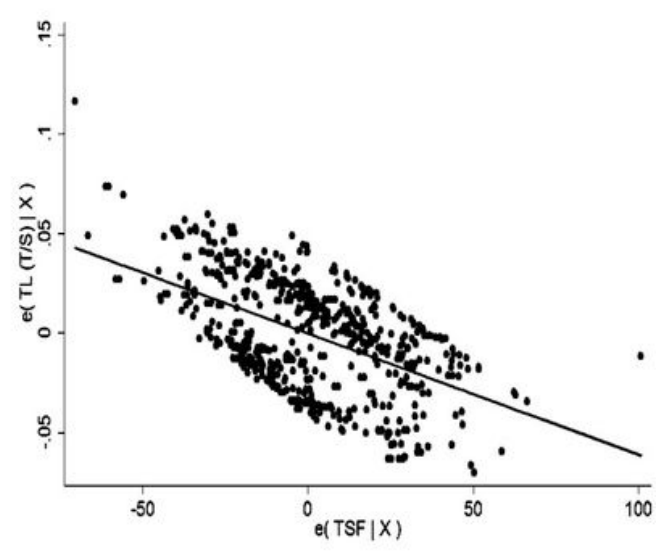

C

Figure 3

Figure 3

Partial correlations between Leukocyte Telomerase Length (LTL) and skinfold thickness [(a)

Subscapular/triceps ratio $(r=-0.4371 ; p=0.0001)$, (b) Central skinfolds $(r=-0.8375 ; p=0.0001)$ and $(c)$ total skinfolds $(\Sigma 6 S F, r=-0.8560 ; p=0.0001)]$ in women with prediabetes $(n, 797)$. Values have been adjusted for age. 


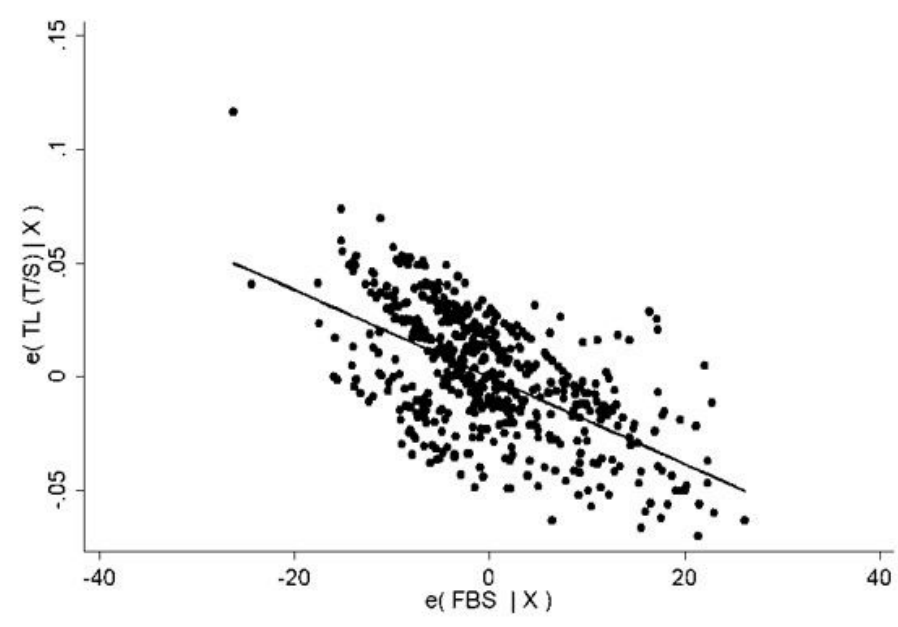

a

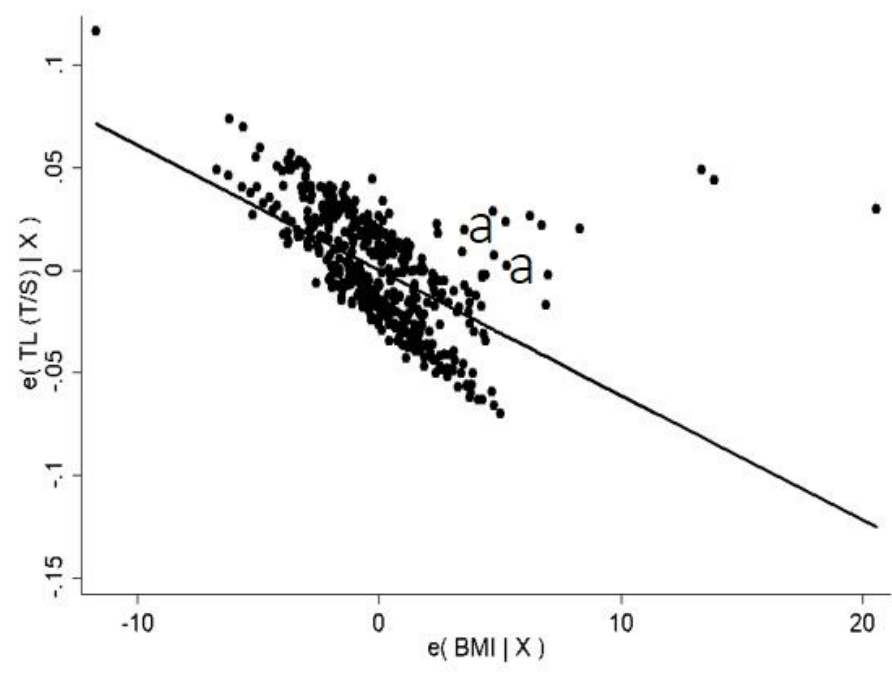

C

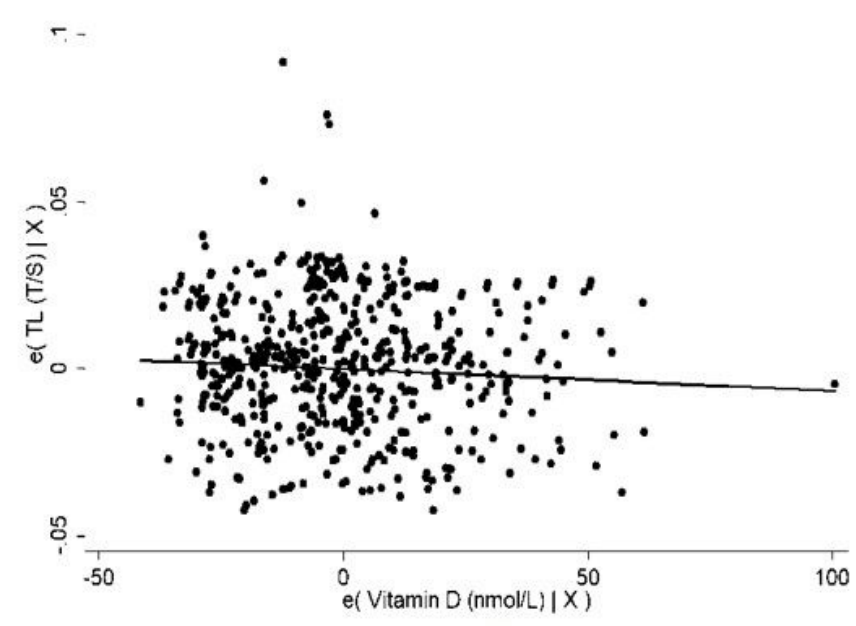

b

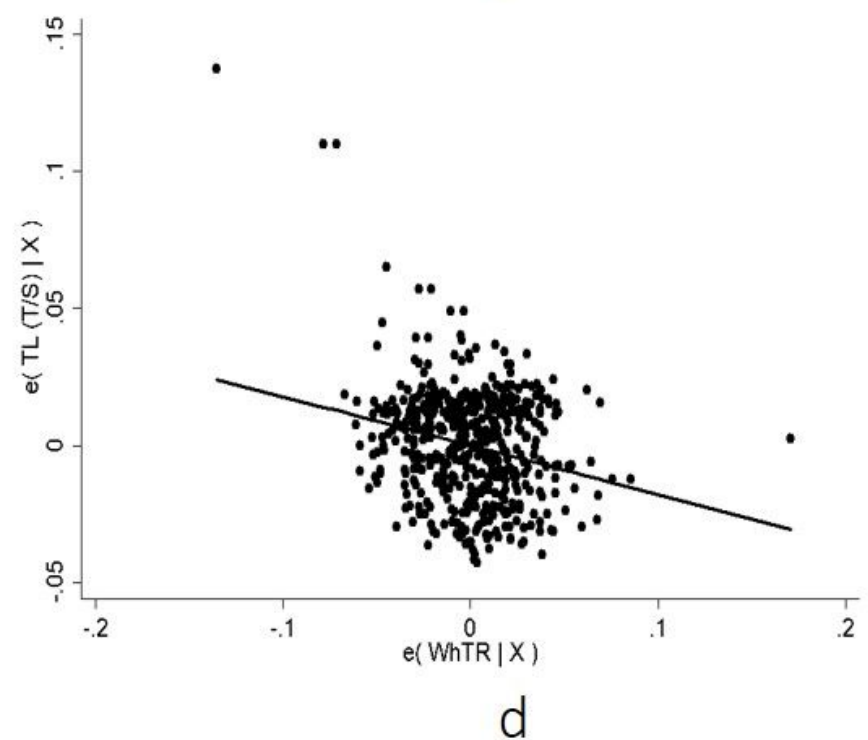

Figure 4

\section{Figure 4}

Partial correlations of Leukocyte Telomerase Length (LTL) and fasting blood glucose (a), LTL and vitamin D (b), LTL and body mass index (c), and LTL and waist-to- height ratio in women with prediabetes ( $\mathrm{n}$, 797). All values have been adjusted for age. 


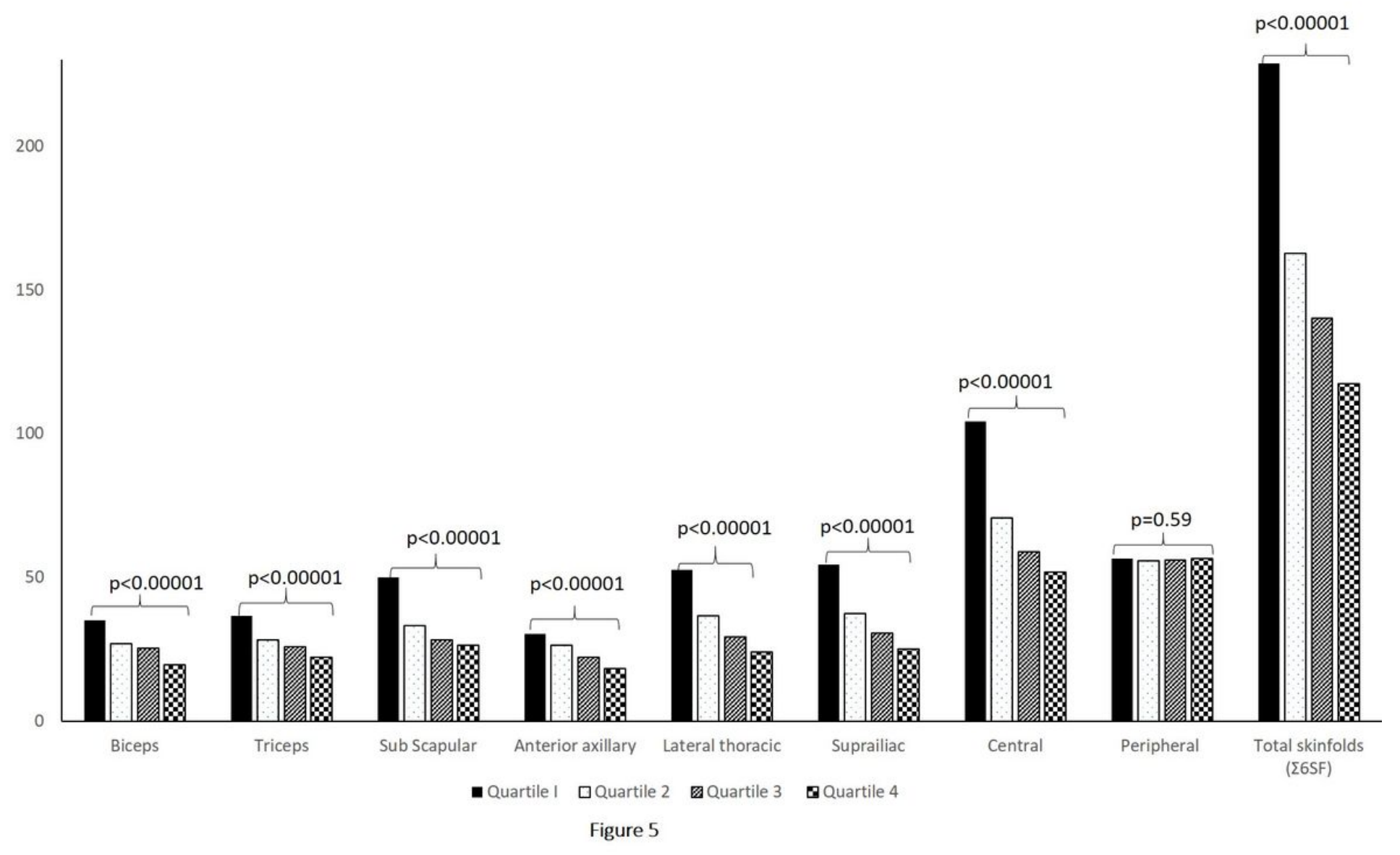

Figure 5

Skinfolds thickness according to quartiles of Leukocyte Telomerase Length 